ISSN 1991-8631

Original Paper

http://indexmedicus.afro.who.int

\title{
Etude de l'effet du mode de production des graines et de la saison de culture sur la montaison prématurée de l'oignon Allium cepa L., variété « Violet de Galmi » au Nord du Burkina Faso
}

\author{
Windpouiré Vianney TARPAGA*, Koussao SOME et M. Larbouga BOURGOU
}

INERA/CMFPT, BP 403 Bobo-Dioulasso, Burkina Faso.

*Auteur correspondant ; E-mail : tarwendp@yahoo.fr; Tel : +22670 708061.

\section{RESUME}

Cette étude visait à évaluer la montaison prématurée de la variété d'oignon Violet de Galmi en fonction de la saison de culture et du mode de production des semences. L'évaluation a été conduite sur des plants d'oignons obtenus à partir de graines issues de cycles de production d'une part annuel et d'autre part bisannuel. L'étude a été conduite en 2007 et 2009, en deux saisons distinctes à savoir la saison précoce à partir d'août et la saison normale à partir d'octobre. L'essai a été disposé en blocs complets randomisés avec quatre répétitions. Les paramètres mesurés ont été le pourcentage de plants ayant manifesté le phénomène et le délai moyen d'émission des hampes florales. On a mesuré en montaison prématurée $99,17 \pm 1,44 \%$ contre $60,00 \pm 9,10 \%$ en 2007 et $94,92 \pm 5,13 \%$ contre $29,35 \pm 4,40 \%$ en 2009 , respectivement pour les graines annuelles et bisannuelles. En saison précoce et normale, le délai d'apparition des hampes a été de 114,64 et 97,67 jours pour les graines annuelles, contre 127 et 112 jours pour les graines bisannuelles. L'étude a montré qu'en saison de culture précoce ou normale, les graines annuelles donnent des plants à montaison plus élevée, dont les hampes apparaissaient plus tôt.

(C) 2013 International Formulae Group. All rights reserved.

Mots clés : Délai de montaison, hampe florale, saison, température, graines bisannuelles.

\section{INTRODUCTION}

L'oignon constitue au plan mondial, le deuxième produit horticole le plus important après la tomate. Il est produit par plus de 175 pays sur environ 3,6 millions d'hectares de terres cultivables (d'Alessandro et Soumah, 2008). L'oignon représente en Afrique de l'Ouest 10 à $25 \%$ de la consommation de légumes des populations (Faivre-Dupaigre et al., 2006). La production d'oignon de cette région demeure cependant déficitaire, entrainant des importations massives, estimée en 2010 à 297188 tonnes, selon les statistiques d'EUROSTAT (Ivanovic, 2011). L'utilisation des semences de mauvaise qualité constitue l'un des obstacles majeurs à la bonne productivité de l'oignon, notamment pour la très répandue variété Violet de Galmi en 
Afrique de l'Ouest. En effet, ce cultivar est très réputé pour sa sensibilité à la montaison prématurée, entrainant de faibles rendements et des productions de mauvaise qualité. Cette montaison prématurée pour les variétés très sensibles peut induire des pertes de rendements bruts pouvant atteindre $73 \%$ sur la variété Granex 33, selon Sanders et Cure (1996).

De nombreux travaux ont montré que plusieurs facteurs tels que la température, la taille des plants, la photopériode, etc. peuvent expliquer la montaison prématurée des variétés d'oignon de jours courts. Selon Brewster (1994) et Sanon (1999), ce phénomène apparaît chaque fois que des plants d'oignon d'un certain âge subissent de basses températures. Pour Sanders et Cure (1996), la montaison prématurée des plants d'oignon se produit, quelle que soit la photopériode, sous une température de 6-12 ${ }^{\circ} \mathrm{C}$. Ces auteurs notent aussi qu'il existe une taille minimale pour chaque variété pour développer des hampes florales. La variabilité de la montaison prématurée en fonction de la taille des plants a été également relevée par Shiraiwa (2008). Par ailleurs, il est ressorti que de basses températures lors de la formation des graines, conduisaient à une augmentation du phénomène de montaison prématurée des plants issus de ces graines (Han et Lee, 1985; Tarpaga et al., 2011). Rouamba (1993) a montré que l'importance de la montaison prématurée dépendait du mode de production des graines. Les graines issues d'un cycle annuel ou méthode « seed to seed » présenteraient une plus grande aptitude à la montaison prématurée que celles issues d'un cycle bisannuel ou méthode «bulb to seed». Ainsi, la pratique paysanne qui consiste à récolter les semences sur des ombelles formées en $1^{\text {er }}$ cycle de culture serait la principale cause d'altération de la qualité génétique des populations améliorées de la variété Violet de Galmi. Le présent travail se propose d'étudier le comportement des graines de Violet de Galmi par rapport à la montaison prématurée, en fonction du mode de production des graines et de la saison de culture. Elle vise à comprendre davantage le phénomène de formation des hampes florales dès la première année de culture à partir des graines, pour les variétés d'oignons de jours courts.

\section{MATERIEL ET METHODES}

L'étude a été conduite en deux (2) essais sur deux campagnes distinctes, à savoir en 2006-2007 et en 2008-2009.

\section{Choix des graines et des saisons de culture}

Deux (2) types de graines d'oignon de la variété Violet de Galmi ont été utilisés au cours des essais, à savoir les graines annuelles et les graines bisannuelles. Les premières sont obtenues à partir d'ombelles de plants issus de semences graines, tandis que les secondes provenaient de plants issus de semences bulbes. Cependant, les lots pour chaque type de graines ont été différents suivant les essais. En 2006-2007, les graines annuelles utilisées ont été récoltées dans des champs paysans et les graines bisannuelles étaient de la semence de la variété $\mathrm{FBO} 1$ produites en station de recherche. Cette variété a été sélectionnée par l'INERA et le CIRAD à partir de populations locales d'oignon Violet de Galmi du Niger. Elle se caractérisait par un taux de montaison prématurée de 10 à $15 \%$ (INERA, 2003). Pour l'essai conduit en 2008-2009, les graines annuelles ont été récoltées en 2007-2008 sur des plants d'oignon FBO1 en montaison prématurée.

Deux saisons de culture ont été choisies pour la conduite des essais. La première saison dont les semis ont été faits à partir de la mi-août a été qualifiée de saison «précoce». La seconde saison a été qualifiée de «saison normale » car elle correspond au calendrier normal de la culture de l'oignon au 
Burkina Faso. Les semis sont pratiqués en début octobre pour cette saison.

\section{Opérations culturales}

L'essai a été conduit suivant un dispositif en blocs complets randomisés avec quatre répétitions. Les parcelles élémentaires mesuraient chacune $4 \mathrm{~m}$ de longueur et $1,20 \mathrm{~m}$ de largeur utile, soit une superficie plantée de $4,8 \mathrm{~m}^{2}$.

Les plants ont d'abord été conduits en pépinières sur le site expérimental, aux dates indiquées en Tableau 1. Les semis de la saison précoce se faisant en période pluvieuse, des dispositions ont été prises pour éviter la destruction des pépinières. On a utilisé à cet effet, des germoirs mesurant chacun $1,2 \mathrm{~m}$ de longueur, 0,6 $\mathrm{m}$ de largeur et $0,1 \mathrm{~m}$ de profondeur. Les semis en saison normale ont été faits en plein-terre. L'aire de semis a été traitée au Diafuran $(5 \mathrm{G})$ et au manèbe $(80 \%)$ pour prévenir les fontes de semis et autres attaques de nuisibles.

Avant le repiquage, un labour à plat a été effectué à l'aide d'une charrue à traction animale, suivi d'une pulvérisation des mottes. Pour la fertilisation, une fumure organique à la dose de $5 \mathrm{~kg} / \mathrm{m}^{2}$ et un engrais minéral N.P.K (14-23-14) à la dose de $90 \mathrm{~g} / \mathrm{m}^{2}$ ont été appliqués au sol. Le repiquage a été effectué en 3 double-lignes par parcelle élémentaire. Des écartements de $0,30 \mathrm{~m}$ entre les doublelignes, de $0,10 \mathrm{~m}$ entre plants situés sur la même ligne et de $0,15 \mathrm{~m}$ entre plants de lignes jumelles ont été adoptés. La densité moyenne de plantation était de 50 plants $/ \mathrm{m}^{2}$. A partir de 30 jours après le repiquage, un apport de 500 $\mathrm{g}$ d'urée par semaine a été fait par fertigation, sur 4 semaines successivement, soit une quantité totale de $2 \mathrm{~kg}$.

L'irrigation des parcelles a été effectuée par un réseau goutte à goutte à basse pression. Elle a été fonction de la saison et du stade phénologique des plants. Les apports d'eau ont été arrêtés pour chaque traitement, au couchage des feuilles d'environ $50 \%$ des plants des parcelles.

\section{Collecte des données}

Les variables observées ont été le pourcentage des plants montés en graines en première année de culture et le délai moyen d'apparition des hampes florales. Pour déterminer ces paramètres, quarante (40) plants ont été identifiés au hasard et étiquetés par traitement et par répétition. Les observations étaient effectuées tous les deux (2) jours. Par ailleurs, la température et l'humidité relative de l'air ont été enregistrées durant toute l'expérimentation un thermo hygromètre, et les différentes moyennes ont été calculées. Les comparaisons statistiques ont porté sur deux facteurs principaux à savoir le cycle de production des graines d'une part, et la saison de culture d'autre part.

Tableau 1 : Etapes de l'itinéraire de production des bulbes en 2007 et 2009.

\begin{tabular}{lcccc}
\hline \multirow{2}{*}{ Paramètres } & \multicolumn{2}{c}{ Essai 2007 } & \multicolumn{2}{c}{ Essai 2009 } \\
\cline { 2 - 5 } & $\begin{array}{c}\text { Saison } \\
\text { précoce }\end{array}$ & $\begin{array}{c}\text { Saison } \\
\text { normale }\end{array}$ & $\begin{array}{c}\text { Saison } \\
\text { précoce }\end{array}$ & $\begin{array}{c}\text { Saison } \\
\text { normale }\end{array}$ \\
\hline Date de semis & $17 / 08 / 06$ & $10 / 10 / 06$ & $29 / 08 / 08$ & $17 / 10 / 08$ \\
Date de repiquage & $04 / 10 / 06$ & $24 / 11 / 06$ & $14 / 10 / 08$ & $03 / 12 / 08$ \\
Durée de pépinière (jour) & 48 & 45 & 46 & 47 \\
Date de récolte & $07 / 02 / 07$ & $30 / 03 / 07$ & $10 / 03 / 09$ & $14 / 04 / 09$ \\
Doses d'irrigation estimée $\left(1 / \mathrm{m}^{2}\right)$ & 580 & 680 & 700 & 740 \\
\hline
\end{tabular}




\section{Analyse des données recueillies}

Les données ont été collectées sur le pourcentage de montaison prématurée et le délai moyen d'émission de la hampe florale. Une analyse des distributions fréquentielles des hampes florales a été réalisée avec le cycle de production des graines comme traitement principal. L'analyse de variance a été faite au seuil de risque alpha de $5 \%$, suivie des tests de comparaison des moyennes selon la procédure de Bonferroni. L'ensemble des traitements statistiques a été effectué à l'aide du programme STATA 10.0 (Statcorp, 2007).

\section{RESULTATS}

Influence du mode de production des graines sur la montaison prématurée

En saison précoce, l'analyse de variance a montré une différence significative entre les graines annuelles et bisannuelles par rapport au taux de montaison prématurée en $2007(\mathrm{P}>\mathrm{F} ; 0,01)$, et très hautement significative en 2009 ( $\mathrm{P}>\mathrm{F} ; 0,00)$, selon le Tableau 2. Quelles que soient l'année et les saisons de culture, les graines annuelles ont donné des plants qui présentaient les taux de montaison prématurée les plus élevés. Ainsi, en 2007, les taux de montaison prématurée des plants étaient de $99,17 \pm 1,44 \%$ pour les graines annuelles, contre $60,00 \pm 9,10 \%$ pour les graines bisannuelles. En 2009, les taux étaient de $94,92 \pm 5,13 \%$ pour les graines annuelles, contre $29,35 \pm 4,40 \%$ pour les graines bisannuelles (Tableau 2).

En saison normale 2007, la différence entre les graines annuelles et bisannuelles n'était par contre pas significative pour la même variable, selon le même tableau. Le taux de montaison prématurée était de 100\% pour les graines annuelles, contre 93,33\% pour les bisannuelles.

Cependant, en 2009, la différence entre les graines annuelles et les graines bisannuelles était significative $(\mathrm{P}>\mathrm{F} ; 0,01)$ par rapport au taux de montaison prématurée. Ce taux était de $94,17 \%$ pour les graines annuelles et $80 \%$ pour les graines bisannuelles.

Par rapport au délai moyen d'émission de la hampe florale, l'analyse de variance a montré une différence significative $(\mathrm{P}>\mathrm{F}$; $0,02)$ entre les graines annuelles et les graines bisannuelles en 2007 et dans les deux saisons de culture. Par contre, la différence entre les deux types de graines était non significative en 2009 (Tableau 3).

En effet, en saison précoce de 2007, les plants issus des graines annuelles ont mis en moyenne 114,67 jours pour émettre une hampe florale contre 127 jours pour les plants issus des graines bisannuelles. En saison normale également, on note que les plants issus des graines annuelles ont mis 97,67 jours à émettre leurs hampes florales, contre 112 jours pour les graines bisannuelles (Tableau $3)$.

\section{Influence de la saison de culture sur la montaison prématurée}

L'examen du Tableau 2 révèle deux (2) tendances caractéristiques pour le taux de montaison prématurée, selon que nous considérions les graines annuelles ou bisannuelles. En effet, la différence entre les deux saisons de cultures n'était pas significative par rapport au taux de montaison prématurée, quelle que soit l'année pour les graines annuelles. Par contre, la saison précoce $(60,00 \pm 9,01 \%) \quad$ a été significativement différente de la saison normale $(93,33 \pm 6,29 \%)$ pour les plants issus des graines bisannuelles en 2007. Il en est de même en 2009, où la différence entre les deux saisons a été hautement significative $(\mathrm{P}>\mathrm{F}$; $0,001)$. On a enregistré un taux de montaison prématurée de $29,35 \pm 4,40 \%$ en saison précoce, contre $80,00 \pm 2,50 \%$ en saison normale pour les plants issus des graines bisannuelles.

Des différences de délais moyens d'apparition des hampes florales des plants ont été notées entre les deux (2) saisons de cultures, aussi bien en 2007 qu'en 2009 et ce, 
quel que soit le type de graines utilisées (Tableau 3). Pour les graines annuelles en 2007, le délai moyen de montaison prématurée en saison précoce $(114,67$ jr) a été supérieur $(\mathrm{P}>\mathrm{F} ; 0,03)$ à celui enregistré en saison normale $(97,67 \mathrm{jr})$. Quand aux graines bisannuelles, la différence a été hautement significative pour les graines bisannuelles ( $>$ F ; 0,004), avec toujours un délai de montaison des plants plus long en saison précoce $(127 \mathrm{jr})$ qu'en saison normale (112 j). En 2009, les différences de délais entre les deux saisons de cultures ont été hautement significatives aussi bien avec les graines annuelles $(\mathrm{P}>\mathrm{F} ; 0,005)$, qu'avec les graines bisannuelles $(\mathrm{P}>\mathrm{F} ; 0,008)$. En effet, on observe au Tableau 3 qu'avec les graines annuelles, le délai de montaison prématurée des plants en saison précoce $(133,82$ jr) a été supérieur à celui des plants cultivés en saison normale $(115,27 \mathrm{jr})$. Avec les graines bisannuelles, la tendance a été la même avec une supériorité du délai de montaison prématurée en saison précoce $(132,33$ jr) par rapport à la saison normale $(116,61 \mathrm{jr})$.

\section{Répartition temporelle des émissions de hampes florales}

L'analyse des distributions des effectifs de plants à hampes florales en année 2007, montre que les émissions de hampes commencent plus tôt avec les graines annuelles dans les deux saisons de cultures (Figures 1 et 2).

Par ailleurs, en saison précoce on observe un décalage de deux semaines entre le mode de la distribution des graines annuelles $(8 / 12 / 06)$ par rapport à celui de la distribution des graines bisannuelles (22/12/06). Cet indicateur de position correspond dans cette étude à la date où il a été enregistré le plus grand nombre d'émission de hampes. Cette date est également précipitée de trois (3) semaines pour les graines annuelles (10/01/07) par rapport aux graines bisannuelles (31/01/07) en saison normale de culture.

En campagne 2009, il ressort que la répartition temporelle des hampes florales pour les deux types de graines a suivi une tendance uniforme, plus marquée cependant en saison normale (Figures 3 et 4). Il apparaît une légère différence d'amplitude entre les courbes des deux distributions en saison précoce (Figure 3). Par ailleurs, on note l'absence d'un décalage prononcé dans le début des apparitions de hampes et dans la position des modes.

Tableau 2 : Taux de montaison prématurée des plants en fonction du mode de production des semences et de la saison de culture en 2007 et 2009.

\begin{tabular}{lccc}
\hline Année & Saison de culture & Types de semences & Taux de montaison (\%) \\
\hline \multirow{2}{*}{2007} & \multirow{2}{*}{ Précoce } & Graines annuelles & $99,17^{\mathrm{a}, \mathrm{c}} \pm 1,44$ \\
\cline { 2 - 4 } & \multirow{2}{*}{ Normale } & Graines bisannuelles & $60,00^{\mathrm{b}, \mathrm{c}} \pm 9,01$ \\
\cline { 2 - 4 } & \multirow{2}{*}{ Précoce } & Graines annuelles & $100,00^{\mathrm{a}, \mathrm{c}} \pm 0,00$ \\
\cline { 3 - 4 } 2009 & Graines bisannuelles & $93,33^{\mathrm{a}, \mathrm{d}} \pm 6,29$ \\
\cline { 3 - 4 } & \multirow{2}{*}{ Normale } & Graines annuelles & $94,92^{\mathrm{a}, \mathrm{c}} \pm 5,13$ \\
\cline { 2 - 4 } & & Graines bisannuelles & $29,35^{\mathrm{b}, \mathrm{c}} \pm 4,40$ \\
\cline { 2 - 4 } & & Graines annuelles & $94,17^{\mathrm{a}, \mathrm{c}} \pm 1,44$ \\
\hline
\end{tabular}

Les comparaisons selon le test de Bonferroni ont été faites par saison (lettres de $1^{\text {er }}$ rang) et par type de graines (lettre de $2^{\text {nd }}$ rang) et les moyennes affectées de la même lettre ne diffèrent pas significativement au seuil de 5\%. 
Tableau 3 : Délais moyens de montaison prématurée des plants en fonction du mode de production des semences et de la saison de culture en 2007 et 2009.

\begin{tabular}{lccc}
\hline Année & Saison de culture & Types de semences & Délais de montaison $(\mathbf{j r})$ \\
\hline \multirow{3}{*}{2007} & \multirow{2}{*}{ Précoce } & Graines annuelles & $114,67^{\mathrm{a}, \mathrm{c}} \pm 2,52$ \\
\cline { 2 - 4 } & & Graines bisannuelles & $127,00^{\mathrm{b}, \mathrm{c}} \pm 1,00$ \\
\cline { 2 - 4 } & \multirow{2}{*}{ Normale } & Graines annuelles & $97,67^{\mathrm{a}, \mathrm{d}} \pm 3,06$ \\
\cline { 3 - 4 } & \multirow{2}{*}{2009} & Graines bisannuelles & $112,00^{\mathrm{b}, \mathrm{d}} \pm 1,00$ \\
\cline { 2 - 4 } & \multirow{2}{*}{ Précoce } & Graines annuelles & $133,82^{\mathrm{a,c}} \pm 3,46$ \\
\cline { 2 - 4 } & \multirow{2}{*}{ Normale } & Graines bisannuelles & $132,33^{\mathrm{a}, \mathrm{c}} \pm 2,02$ \\
\cline { 2 - 4 } & & Graines annuelles & $115,27^{\mathrm{a,d}} \pm 1,79$ \\
\cline { 2 - 4 } & & Graines bisannuelles & $116,61^{\mathrm{a,d}} \pm 1,03$ \\
\hline
\end{tabular}

$\overline{\text { Les comparaisons selon le test de Bonferroni ont été faites par saison (lettres de } 1^{\text {er }} \text { rang) et par type de graines (lettre de } 2^{\text {nd }}}$ rang) et les moyennes affectées de la même lettre ne diffèrent pas significativement au seuil de 5\%.

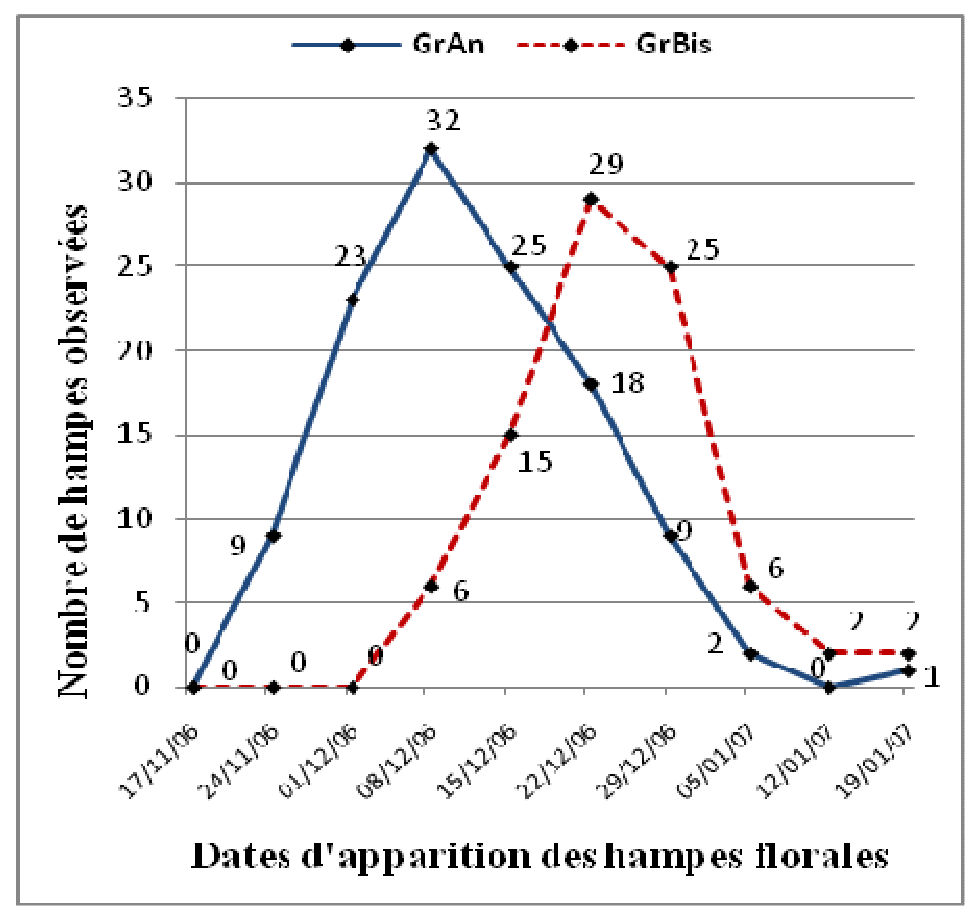

Figure 1 : Etalement du nombre moyen de hampes selon la date d'apparition en saison précoce 2007. 


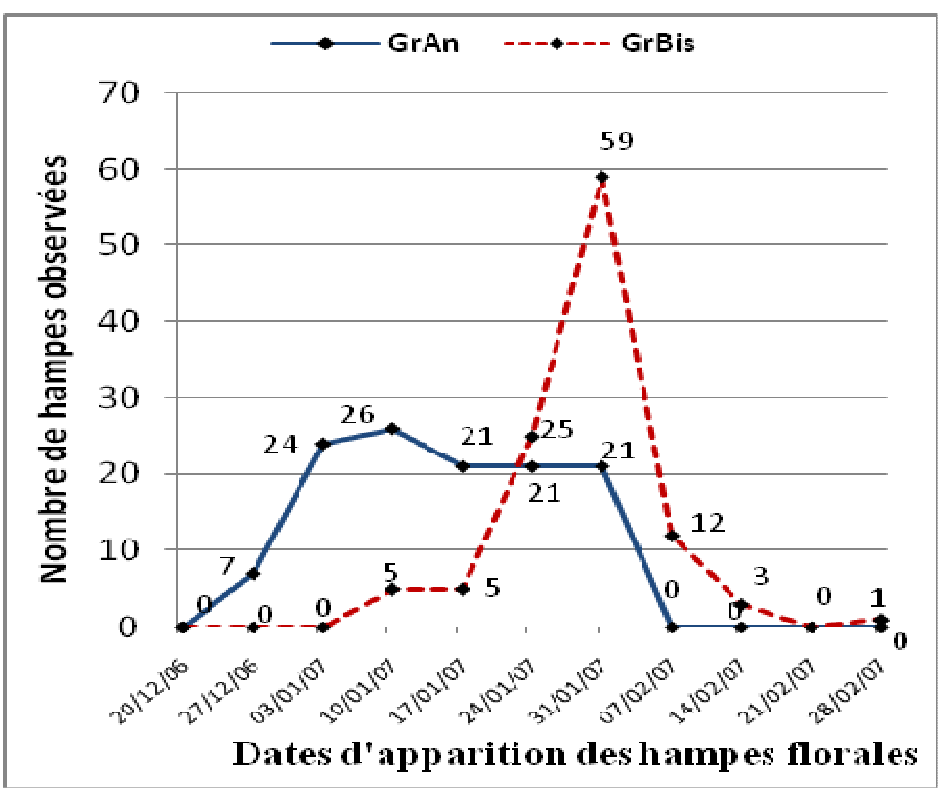

GrAn : Graines annuelles; GrBis : Graines bisannuelles

Figure 2 : Etalement du nombre moyen de hampes selon la date d'apparition en saison normale 2007.

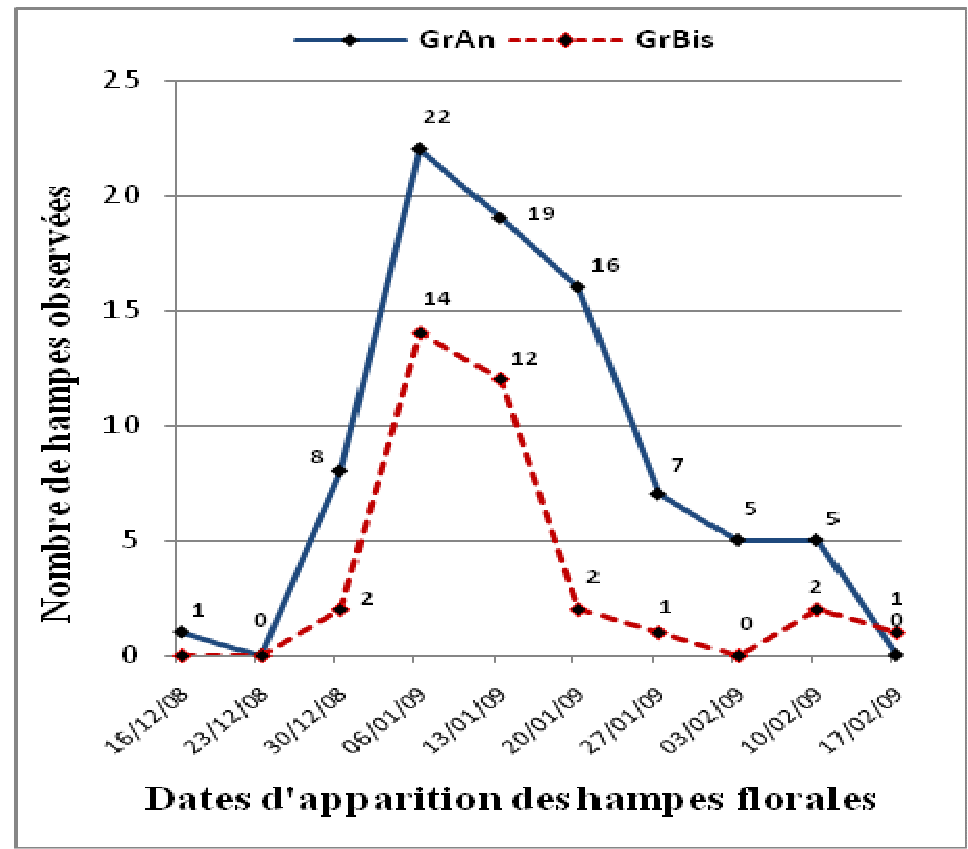

Figure 3 : Etalement du nombre moyen de hampes selon la date d'apparition en saison précoce 2009. 


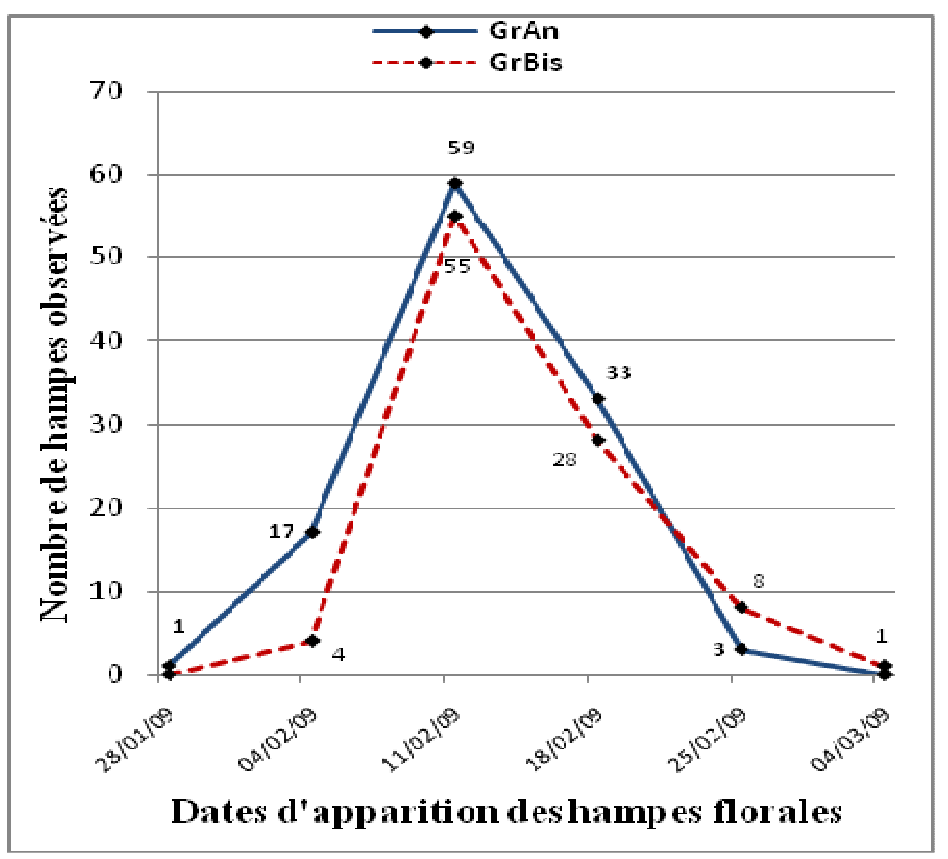

GrAn : Graines annuelles; GrBis : Graines bisannuelles

Figure 4 : Etalement du nombre moyen de hampes selon la date d'apparition en saison normale 2009.

\section{DISCUSSION}

Mode de production des graines et montaison prématurée

Les graines annuelles diffèrent fondamentalement des graines bisannuelles par le fait que les plants qu'elles produisent présentent une plus grande sensibilité à la montaison prématurée. En effet, on a enregistré pour les plants issus de ce type de graines, un pourcentage de hampes florales plus élevé dans tous les essais, sauf en saison normale 2007. En effet, pour cet essai, bien que numériquement le pourcentage de montaison prématurée des plants de graines annuelles était supérieur, la différence statistique n'était pas significative. La sensibilité des plants issus de ces graines semble se confirmer par leur relative rapidité à émettre une hampe florale. En effet, on note le raccourcissement d'environ deux (2) semaines par rapport aux plants de graines bisannuelles, du délai moyen d'apparition de la hampe en
2007. Cependant, les comparaisons de 2009 ne permettent pas d'aboutir aux mêmes conclusions car en saison précoce, l'apparition des hampes florales des plants de graines annuelles se prolonge dans les mêmes délais que les plants de graines bisannuelles. Mais cette émission de hampes florales intervenait à des taux très élevés dans le cas des plants de graines annuelles. Ce comportement des graines annuelles n'a pu être expliqué dans cette étude et nécessite des investigations supplémentaires.

\section{Saison de culture et montaison prématurée}

Dans les deux (2) saisons de culture, les plants des graines annuelles présentent le même taux d'émission de hampes florales, tandis que les plants de graines bisannuelles en saison précoce manifestent très peu le phénomène.

La différence de température entre les deux saisons de culture serait à l'origine de ce comportement des graines. En effet, selon les 
travaux de Brewster (1983), il existe une température minimale efficace pour induire l'apparition de la hampe florale. La même étude précise qu'il faut une exposition des plants à cette température efficace pendant une certaine durée pour avoir une réponse. En saison précoce comme en saison normale, les moyennes des températures minimale en 2007 $\left(17,33^{\circ} \mathrm{C}\right.$ et $\left.14,17^{\circ} \mathrm{C}\right)$ et en $2009\left(15,57^{\circ} \mathrm{C}\right.$ et $12,54{ }^{\circ} \mathrm{C}$ ) auraient été suffisamment basses pour induire la formation de hampes florales, mais différemment selon le type de graines.

Les graines annuelles auraient un seuil thermique (somme de basses températures) moins élevé pour émettre leurs hampes florales. Cette éventualité justifierait que la quasi-totalité de la population de plants issus de graines annuelles émette une hampe florale en saison précoce, bien que les températures y sont moins basses qu'en saison normale. Quant aux plants issus des graines bisannuelles, elles exigeraient des températures plus basses que celles de la saison précoce pour émettre des hampes florales. Ainsi, les températures de la saison normale seraient plus favorables à ces plants. Les quelques plants issus de graines bisannuelles qui ont émis une hampe florale en saison précoce seraient de résistance moindre à la montaison prématurée. L'explication serait liée à l'hétérogénéité génétique de la semence d'oignon. Il est en effet bien connu, qu'en raison de la forte allogamie de l'oignon, il est très difficile de maintenir la pureté des semences, surtout pour ce caractère de montaison prématurée. D'Arondel De Hayes et Traoré (1985), de même que Currah et Proctor (1993) l'ont bien mentionné. Les graines bisannuelles apparaissent donc plus tolérantes au phénomène de montaison prématurée, d'où les recommandations de recourir à ce type de semences pour la production de bulbes.

La persistance de la montaison prématurée des variétés sensibles d'oignon serait lié à un caractère primitif de la plante. En effet, l'oignon à l'origine serait à cycle annuel. Il aurait été domestiqué et rendu bisannuel pour les besoins de l'homme. Ainsi, la plante aurait tendance à retourner à l'état primitif, si les conditions environnementales sont favorables.

Quant aux délais moyens d'émission des hampes florales, on constate qu'entre la saison précoce et la saison normale, il y a un décalage en moyenne de deux semaines quel que soit le type de graine et l'année de l'essai. Une explication serait que plus les températures sont basses en dessous du seuil (minima) critique, plus vite la somme des basses températures serait atteinte pour déclencher le phénomène. Cela prouve que le Violet de Galmi, comme beaucoup d'autres variétés d'oignon, est sensible au thermopériodisme. Ce comportement des plantes confirment donc les résultats de Brewster (1983 et 1985) et les observations en Côte d'Ivoire rapportées par Currah et Proctor (1993) sur l'exigence d'un besoin de froid des plants d'oignon pour déclencher l'émission de la hampe florale. Ce besoin qui est exprimé en nombre d'heures d'exposition des plants aux basses températures, serait variable selon l'âge, la taille de la plante, le cultivar, la photopériode, etc. (Brewster, 1985 ; Sanders et Cure, 1996).

\section{Répartition temporelle des émissions de hampes florales}

La sensibilité des graines annuelles à la montaison prématurée transparaît de nouveau à travers l'analyse des distributions des effectifs des plants à hampes florales. La première hampe pour ce type de graines en saison précoce est apparue 35 jours plus tôt (le 3/11/06) par rapport au premier plant issu d'une graine bisannuelle (le 8/12/06). Par ailleurs, le pic des effectifs est également atteint deux (2) ou trois (3) semaines plus tôt, comparé aux plants des graines bisannuelles. Leur moindre exigence en quantité de froid ferait qu'elles aient vite atteint le seuil critique pour émettre une hampe florale. En 2009, le comportement des plants traduit plutôt une ressemblance entre ces deux types de graines. Cependant, la grande amplitude de la 
distribution des hampes en saison précoce confirme bien que les graines bisannuelles résistent mieux au phénomène. En saison normale par contre, cette résistance semble céder sous l'effet de la basse température $\left(12,5^{\circ} \mathrm{C}\right)$.

\section{Conclusion}

La pratique qui consiste à utiliser les graines annuelles pour la production des bulbes d'oignon est encore très répandue en Afrique de l'Ouest. A travers cette étude, il apparait que ce type de graines présente une très grande sensibilité à la montaison prématurée. Ce phénomène concerne parfois la quasi-totalité de la population des plants en culture. Ces résultats confirment bien le fait que la méthode de production «seed to seed » n'est pas appropriée sous les conditions climatiques locales.

Les basses températures apparaissent comme étant le principal facteur d'induction de hampes florales et les graines annuelles semblent avoir des seuils thermiques plus bas que les graines bisannuelles. En conditions fraîches, les graines bisannuelles présentent une meilleure résistance à la montaison prématurée. Cependant, ces dernières admettent aussi un taux minimal de hampes florales, sans doute lié à l'état physiologique des graines ou à leur hétérogénéité génétique. En effet, le mode de reproduction allogame de l'oignon est une des contraintes au maintien de la pureté génétique des variétés développées.

Néanmoins, de très faibles taux de montaison sont accessibles dans des conditions strictes de multiplication des graines d'oignon. Ainsi, il est vivement recommandé aux producteurs pour une culture rentable des bulbes d'oignon, de recourir aux semences sélectionnées, surtout en saisons de culture très fraîches.

\section{REMERCIEMENTS}

Les auteurs remercient sincèrement les Professeurs TAMINI Z., YATTI K J. et ROUAMBA A. pour les corrections apportées au manuscrit. Ils témoignent leurs vives reconnaissances à la Coopération Suisse (DDC) au Burkina Faso qui, à travers son programme « Développement Rural »a assuré le financement et l'appui logistique qui ont permis la réalisation de cette étude.

\section{REFERENCES}

Brewster JL. 1983. Effect of photoperiod, nitrogen nutrition and temperature on inflorescence initiation and development in onion (Allium cepa L.). Annals of Botany, 51: 429-440.

Brewster JL. 1985. The influence of seedling size and carbohydrate status and photon flux density during vernalization on inflorescence initiation in onion (Allium cepa L.). Annals of Botany, 55: 403-414.

Brewster JL. 1994. Onions and Other Vegetable Alliums. Crop Production Science in Horticulture, CABI: Wallingford, UK.

Currah L, Proctor FJ. 1993. La Culture et la Conservation des Oignons sous les Tropiques. CTA: Wageningen, PaysBas.

D'Alessandro S, Soumah A. 2008. Évaluation sous-régionale de la chaîne de valeurs oignon/échalote en Afrique de l'Ouest. Bethesda, MD: projet ATP, Abt Associates Inc.

D’Arondel De Hays J, Traoré G. 1985. Travaux sur l'Oignon en Zone Tropicale Sèche. Etude au Burkina Faso en Zone Sudano-sahélienne. CIRAD-IRAT: Burkina Faso ; 32 p.

Faivre-Dupaigre B, Baris P, Liagre L. 2006. Rapport de l'étude sur la compétitivité des filières agricoles dans l'espace UEMOA. IRAM.

Han GS, Lee KH. 1985. The effect of low temperature treatment at the different ripening stages of radish (Raphanus sativus L.) seeds on bolting. J. Kor. Soc. Hort. Sci., 26: 1-6.

INERA, 2003. Fiche descriptive oignon: Violet de Galmi (12 BF). CMFPT: Burkina Faso ; 2 p. 
Ivanovic D. 2011. Exportation d'oignons hollandaise vers l'Afrique de l'Ouest de 2001-2010. Présentation des données en provenance d'EUROSTAT. Hub Rural.

Rouamba A. 1993. Analyse conjointe par les marqueurs agro-morphologiques et les allozymes de la diversité génétique des populations d'oignons (Allium cepa L.) d'Afrique de l'Ouest. Thèse de Doctorat unique, Université Pierre et Marie Curie, Paris 6, p. 141.

Sanders DC, Cure JC. 1996. Control of bolting in Autumn-sown sweet Onions through undercutting. J. Am. Soc. Hortic. Sci., 121 : 1147-1151.

Sanon M. 1999. Optimisation de l'irrigation à la parcelle par radio-thermométrie, Application à une culture d'oignon
(Allium cepa L.) en climat sahélien (nordouest du Burkina Faso). Thèse de Doctorat unique, ENSA de Renne, p.266.

Shiraiwa N. 2008. Physiological studies on the bolting control for stable production of early summer harvest in bunching onion (Allium fistulosum L.). Special Bulletin of the Tottori Horticultural Experiment Station, 11: 1-92

Statcorp. 2007. Stata Statistical Software: Release 10. College Station, TX, StataCorp., p.148.

Tarpaga WV, Rouamba A, Tamini Z. 2011. Effects of seasons of bulb and seed production on the early bolting of onion (Allium cepa L.) cv 'violet de Galmi'. Journal of Applied Biosciences, 40: 2652-2658. 